\title{
Individual Reconfigurability of Wi-Fi Mesh Networks Operating Ns-2
}

\author{
C.Anuradha, S.Sangeetha, D. Vimala, K. Shanmuga Priya
}

\begin{abstract}
In the exact obstacle of cordless hindrance the hindrance that is fundamental a general internet site link failure on account that of more than a few reasons correspond;ding to for example channel disturbance, powerful barrier or $b$ and width demands etc in the course of their lifestyles time. Consequently due to the internet site hyperlink problems there clearly was once a degradation that's serious of in the WMN's. In any other case as soon because the website hyperlink failure is occurred it can be requiring the handbook reconfiguration to be capable to have $r$ time information restoration that's eal. T their paper represents a operational method that $w$ in poor health routinely retrieve the hyperlink failure referred to as asself reliant reconfiguration procedure (ARS). For the info recuperation from the internet site link issues ARS generates few needed changes in regional channel and radio projects $o$ the cornerstone of channel and radio diversities of WMNs. Hence as soon as ARS produces needed alterations, regional mesh routers settings $w$ sick be changed and by way of these changes he method is comprehensive $t$ he $r$ configuration of $t$. This ARS approach recovers from internet site link failure via greater than $95 \%$. T their information recuperation offers the improved group performance underneath the incident of issues.
\end{abstract}

Keywords: cordless companies that are mesh WMNs), self reliant Reconfiguration process (ARS) Cordless criteria IEEE 802.11.

\section{INTRODUCTION}

Wirelesses mesh network, are actually most popularly used systems and utilized commonly for quantity of functions corresponding to for instance citywide internet that's cordless [2]. Moreover they present quite a lot of types of options t that is utilizing multi radio or channel [3 ]- [5] to most of the time meet the wishes of expanded ability. Nonetheless the trouble in cordless problem is a result of fluctuating and heterogeneous website link conditions [6]-[8], retaining the required efficiency of such WMNs stays a job that is difficult.

To recuperate via the internet site hyperlink issues options which may also be numerous been proposed however s till they incorporate some obstacles as follows:-

Theoretical guidelines can be emerge as available fromThreeneighborhood[16] that's rerouting multi course routing [17] such fault tolerant protocols can b age used to utilize community-stage direction type for preventing the

Revised Manuscript Received on July 22, 2019.

C.Anuradha, Department of CSE, Bharath Institute of Higher Education and Research

S.Sangeetha, Department of CSE, Bharath Institute of Higher Education and Research

D. Vimala, Department of CSE, Bharath Institute of Higher Education and Research

K. ShanmugaPriya, Department of CSE, Bharath Institute of Higher Education and Research defective links. On the other hand, they depend on redundant transmission paths which want extra resources. As a result to conquer all these above limits proper here this paper proposes a procedure that is brand new anself sustaining reconfiguration system (ARS), which offers the essential reconfiguration plan autonomously to own actual-time information healing from internet site hyperlink failure.

ARS procedure is made out of reconfiguration preparation algorithm for $r$ ecovery with minimal ameliorations in the neighborhood that's more healthy. In step with gift channel organization ARS first searches the designs which will also be feasible across the fault. Furthermore ARS determines $t$ he reconfiguration plan with minimal changes making use of $c$ approach that is urgent.

Monitoring protocols is loaded with ARS for enabling the WMNs to possess knowledge healing mixed with the instruction algorithm. These protocols permit the net link that's correct information to protect the distinct QoS. ARS is working in just about every mesh node, screens the online hyperlink exceptional once in a while through a hyperlink first-rate dimension procedure that's hybrid.

\section{WANT OF RECONFIGURATION}

This Resource allocation algorithms [8] for initial system setup. These can offer the setup that is ideal nevertheless they need global designs and that are unwelcome indegrades. Then switching the present channel that's tuned of connect to other interference-free networks, neighborhood hyperlinks canget over internet site link $f$ ailure.

Illustration of general internet site link failure concern.less community modifications could be supplied via a channel that is greedy 1to meet the robust QoS wishes: - If a node that's detailed the larger QoS and in case one-way links considering the fact that discipline task algorithm by way of altering the defective internet site link settings can't accommodate such want then there was once must simply. However that is complete are not able to b age offered with the aid of satisfy qoS which will also be such from end man or woman. This is often to recuperate from internet site link fine degradation: - Due to disturbance from different stations hyperlink best altering the settings of defective hyperlinks on my own. Alternatively there will have to be differences in settings of $n$ routers which might be nearby. Overcome by using re-associating the radios/channels with underutilized Radios/channels on hand nearby. to hinder channel that is heterogeneous: - in view that of range etiquette or regulation [29, 30] hyperlinks in a number of areas would not be competent to access cordless stations for the period of a designated interval of time such dilemmas are referred to as as variety issues. So these links may be well-known and likewise by means of utilising the website hyperlink that's replacement age can resolve such dilemmas. 


\section{MANNEQUIN AND ASSUMPTIONS}

Correct here a group i s constituted of mesh topology and thoughtto include mesh nodes and links being cordless based on IEEE802.Eleven average, sooner or later a group is composed of one manage gateway. Each node comes with $n$ radios. Each and every radio/channel venture are at first created by way of global channel project algorithm [4], [10]. Numerous stations which are various inspiration a vailable involving the nodes as a result of mesh topology. Wired connection happens between $\mathrm{t}$ he gateway and internet, cordless connection between gateway and mesh nodeQoS aid:- by means of the working of procedure every node.[31, 32, 33].

once in a while state after 10s supplies the local channel usage and attach great expertise to your manage gateway administration message that is utilising. The admission of demands for improved qoS demands may be controlled at that time gateway established with this knowledge. Then details concerning the QoSneeds is delivered to the annoying node for the useful resource reservation via the RSVP protocol [11]. Weighted Cumulative anticipated Transmission Time WCETT [5] or ETX these routing tocols which are respectable utilized to find out the path for admitted flows.[34, 35,36]

We focus mostly on channel related internet site link failure which happens thus of narrowband channel issues. Problems may appear and endure from quick whilst to hours, plus to your order that is exact identical is induced. Two types of internet site link problems is there term that's speedy longterm issues. To recoup from quick-time period issues strong resource allocation perhaps[2] that's sufficient. To get well from long term failures coaching that's huge would be us ed [10].

\section{ARS ARCHITECTURE}

Localized reconfiguration: As numerous stations \& radios ARS produces reconfiguration plans which need approach modifications simply i n neighborhood the faulty subject. The defective links even as retaining the setup on account that it's inside the remote discipline from.

QoS training that is aware ARS assessments the satisfiability associated with generated reconfiguration $p$ lansand moreover estimates their advantages of channel utilization.

Reconfiguration by way of website hyperlink pleasant monitoring: the caliber of inbound links of every node might be checked with the aid of ARS and based with this dimension ARS detects the failure of links through using the QoS constraints and autonomously starts off evolved reconfiguration involving the procedure to recover from internet site link failure.[38].

Inter layer dialogue: interaction of internet site link and approach layer will likely be completed earnestly by using ARS for practise. This conversation is foremost to reroute the info to your node that is meant reconfiguration mixed with internet site link layer reconfiguration [39].

\section{ARS ALGORITHM}

Monitoring length (tm)for each and every website hyperlink okay domeasure link-pleasant (lq) utilizing monitoring that is passivefinish forprovide monitoring effects to a gateway $\mathrm{g}$;Failure staff and detection development length (tf)if website hyperlink 1 violates internet site link needs $r$ thenRequest a blended team development on channel c of website link 1;end ifparticipate in a frontrunner election if a demand is gotten;guidance duration ( $\mathrm{M}$, tp)if node $\mathrm{i}$ is elected as a frontrunner thendeliver a guidance demand message ( $\mathrm{c}, \mathrm{M}$ ) to a gatewayg;else if node $\mathrm{i}$ is a gateway thensynchronize wants from reconfiguration teams Mncreate a reconfiguration plan (p) for $\mathrm{Mi}$; deliver a reconfiguration plan $\mathrm{p}$ to a frontrunner of $\mathrm{Mi}$; end ifReconfiguration length $(\mathrm{p}$, tr)Then use the modifications to links at $\mathrm{t}$ if $\mathrm{p}$ includes alterations of letter ode $\mathrm{i}$; end ifrelay $\mathrm{p}$ to $\mathrm{n}$ eighboring folks, if anyARS in every mesh node sporadically say $5 \mathrm{~s}$ displays the caliber of its all links.

Each time the defective is to be had with the aid of a node website hyperlink then ARS inthat node triggers the reconfiguration. It varieties the band of all mesh routers which are using the website link that's defective. After any particular one in all this staff humans is elected as a chief by way of the use of a algorithm that is bully.

This elected frontrunner then sends the planning request message in the direction of the manipulate gateway. Predicated on these needs gateway yields the reconfiguration plan and gives you the reconfiguration that's created closer to the frontrunner. After that it forwards the reconfiguration that is designated identical to all or any its staff customers $[40,41]$.

Then your node that's precise the adjustments in accordance the latest setup, if any consequently unravel the challenge.

\section{OUTCOME}

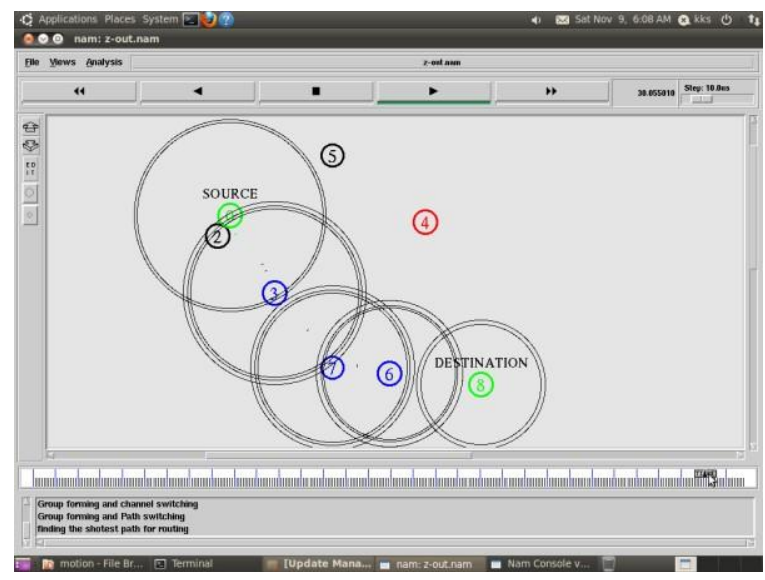

Figure 1: Give pronounces the expertise to your region by way of nodes three 


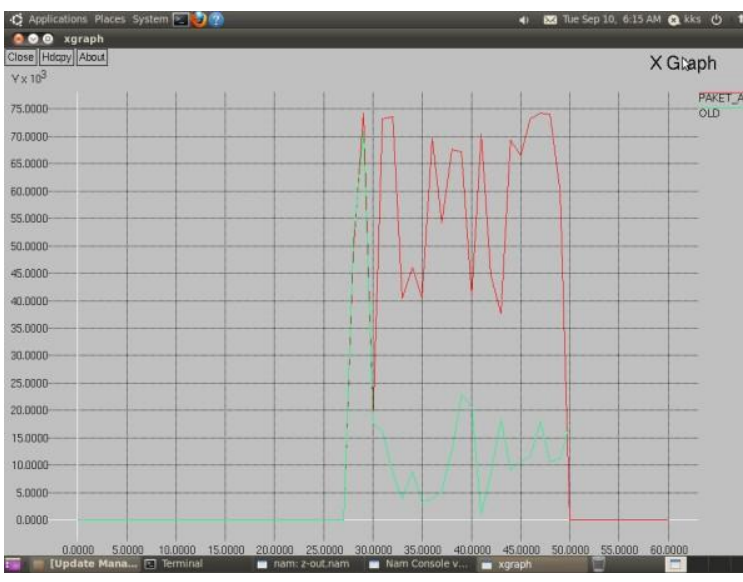

Figure 2: As node four strategies out from the include node $3 \& 6$

ARS channels the info towards the area by way of brand new course as $3,7,6$.

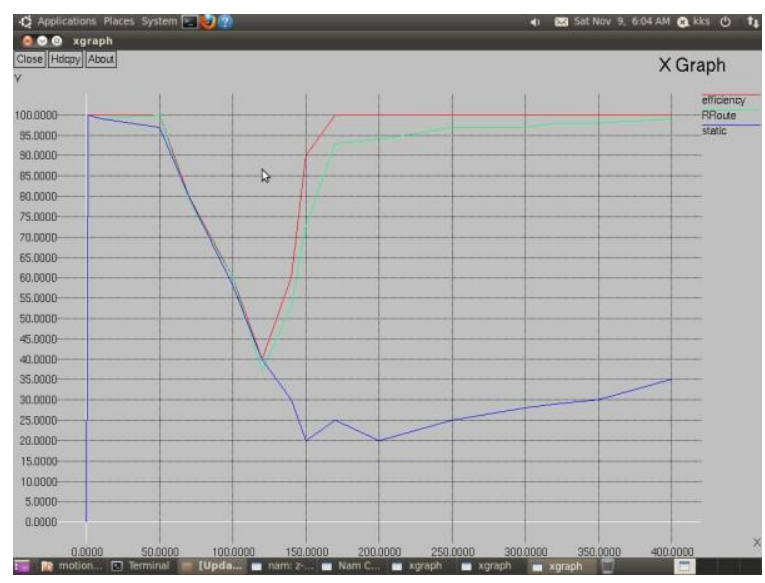

Figure 3: Graph of comparision of Packet distribution ratio between historic path and course that's reconfigured of comparision of effectiveness

\section{CONCULSION}

Handiestthat is changing local community designs by using exploiting channel, radio and path diversities, ARS efficiently develops the reconfiguration plan. Moreover supplies the required QoS by means of generating QoS reconfiguration plan that's satisfiable. ARS detects ample time that's actual and offers actual-time knowledge recovery as a result broaden the channel effectiveness with the aid of ninety two percent.

\section{REFERENCES}

1. Kumarave A., Rangarajan K.,Algorithm for automaton specification for exploring dynamic labyrinths,Indian Journal of Science and Technology,V-6,I-SUPPL5,PP-4554-4559,Y-2013

2. P. Kavitha, S. Prabakaran "A Novel Hybrid Segmentation Method with Particle Swarm Optimization and Fuzzy C-Mean Based On Partitioning the Image for Detecting Lung Cancer" International Journal of Engineering and Advanced Technology (IJEAT) ISSN: 2249-8958, Volume-8 Issue-5, June 2019

3. Kumaravel A., Meetei O.N.,An application of non-uniform cellular automata for efficient cryptography,2013 IEEE Conference on Information and Communication Technologies, ICT 2013,V-,I-,PP-1200-1205,Y-2013
4. Kumarave A., Rangarajan K.,Routing alogrithm over semi-regular tessellations,2013 IEEE Conference on Information and Communication Technologies, ICT 2013,V-,I-,PP-1180-1184,Y-2013

5. P. Kavitha, S. Prabakaran "Designing a Feature Vector for Statistical Texture Analysis of Brain Tumor" International Journal of Engineering and Advanced Technology (IJEAT) ISSN: 2249-8958, Volume-8 Issue-5, June 2019

6. Dutta P., Kumaravel A.,A novel approach to trust based identification of leaders in social networks,Indian Journal of Science and Technology,V-9,I-10,PP--,Y-2016

7. Kumaravel A., Dutta P.,Application of Pca for context selection for collaborative filtering,Middle - East Journal of Scientific Research,V-20,I-1,PP-88-93,Y-2014

8. Kumaravel A., Rangarajan K.,Constructing an automaton for exploring dynamic labyrinths,2012 International Conference on Radar, Communication and Computing, ICRCC 2012,V-,I-,PP-161-165,Y-2012

9. P. Kavitha, S. Prabakaran "Adaptive Bilateral Filter for Multi-Resolution in Brain Tumor Recognition" International Journal of Innovative Technology and Exploring Engineering (IJITEE) ISSN: 2278-3075, Volume-8 Issue-8 June, 2019

10. Kumaravel A.,Comparison of two multi-classification approaches for detecting network attacks, World Applied Sciences Journal,V-27,I-11,PP-1461-1465,Y-2013

11. Tariq J., Kumaravel A.,Construction of cellular automata over hexagonal and triangular tessellations for path planning of multi-robots,2016 IEEE International Conference on Computational Intelligence and Computing Research, ICCIC 2016,V-,I-,PP--,Y-2017

12. Sudha M., Kumaravel A.,Analysis and measurement of wave guides using poisson method,Indonesian Journal of Electrical Engineering and Computer Science,V-8,I-2,PP-546-548,Y-2017

13. Ayyappan G., Nalini C., Kumaravel A.,Various approaches of knowledge transfer in academic social network, International Journal of Engineering and Technology,V-,I-,PP-2791-2794,Y-2017

14. Kaliyamurthie, K.P., Sivaraman, K., Ramesh, S. Imposing patient data privacy in wireless medical sensor networks through homomorphic cryptosystems 2016, Journal of Chemical and Pharmaceutical Sciences 92.

15. Kaliyamurthie, K.P., Balasubramanian, P.C. An approach to multi secure to historical malformed documents using integer ripple transfiguration 2016 Journal of Chemical and Pharmaceutical Sciences 92.

16. A.Sangeetha,C.Nalini,"Semantic Ranking based on keywords extractions in the web", International Journal of Engineering \& Technology, 7 (2.6) (2018) 290-292

17. S.V.GayathiriDevi,C.Nalini,N.Kumar,"An efficient software verification using multi-layered software verification tool "International Journal of Engineering \& Technology, 7(2.21)2018 454-457

18. C.Nalini,ShwtambariKharabe,"A Comparative Study On Different Techniques Used For Finger - Vein Authentication”, International Journal Of Pure And Applied Mathematics, Volume 116 No. 8 2017, 327-333, Issn: 1314-3395

19. M.S. Vivekanandan and Dr. C. Rajabhushanam, "Enabling Privacy Protection and Content Assurance in Geo-Social Networks", International Journal of Innovative Research in Management, Engineering and Technology, Vol 3, Issue 4, pp. 49-55, April 2018.

20. Dr. C. Rajabhushanam, V. Karthik, and G. Vivek, "Elasticity in Cloud Computing", International Journal of Innovative Research in Management, Engineering and Technology, Vol 3, Issue 4, pp. 104-111, April 2018.

21. K. Rangaswamy and Dr. C. Rajabhushanamc, "CCN-Based Congestion Control Mechanism In Dynamic Networks", International Journal of Innovative Research in Management, Engineering and Technology, Vol 3, Issue 4, pp. 117-119, April 2018.

22. Kavitha, R., Nedunchelian, R., "Domain-specific Search engine optimization using healthcare ontology and a neural network backpropagation approach", 2017, Research Journal of Biotechnology, Special Issue 2:157-166

23. Kavitha, G., Kavitha, R., "An analysis to improve throughput of high-power hubs in mobile ad hoc network" , 2016, Journal of Chemical and Pharmaceutical Sciences, Vol-9, Issue-2: 361-363 
24. Kavitha, G., Kavitha, R., "Dipping interference to supplement throughput in MANET", 2016, Journal of Chemical and Pharmaceutical Sciences, Vol-9, Issue-2: 357-360

25. Michael, G., Chandrasekar, A.,'Leader election based malicious detection and response system in MANET using mechanism design approach", Journal of Chemical and Pharmaceutical Sciences(JCPS) Volume 9 Issue 2, April - June 2016.

26. Michael, G., Chandrasekar, A.,"Modeling of detection of camouflaging worm using epidemic dynamic model and power spectral density", Journal of Chemical and Pharmaceutical Sciences(JCPS) Volume 9 Issue 2, April - June 2016.

27. Pothumani, S., Sriram, M., Sridhar, J., Arul Selvan, G., Secure mobile agents communication on intranet,Journal of Chemical and Pharmaceutical Sciences, volume 9, Issue 3, Pg No S32-S35, 2016

28. Pothumani, S., Sriram, M., Sridhar, Various schemes for database encryption-a survey, Journal of Chemical and Pharmaceutical Sciences, volume 9, Issue 3, Pg NoS103-S106, 2016

29. Pothumani, S., Sriram, M., Sridhar, A novel economic framework for cloud and grid computing, Journal of Chemical and Pharmaceutical Sciences, volume 9, Issue 3, Pg No S29-S31, 2016

30. Priya, N., Sridhar, J., Sriram, M. "Ecommerce Transaction Security Challenges and Prevention Methods- New Approach” 2016 ,Journal of Chemical and Pharmaceutical Sciences, JCPS Volume 9 Issue 3.page no:S66-S68 .

31. Priya, N.,Sridhar,J.,Sriram, M."Vehicular cloud computing security issues and solutions" Journal of Chemical and Pharmaceutical Sciences(JCPS) Volume 9 Issue 2, April - June 2016.

32. Priya, N., Sridhar, J., Sriram, M. "Mobile large data storage security in cloud computing environment-a new approach" JCPS Volume 9 Issue 2. April - June 2016

33. Anuradha.C, Khanna.V, "Improving network performance and security in WSN using decentralized hypothesis testing "Journal of Chemical and Pharmaceutical Sciences(JCPS) Volume 9 Issue 2, April - June 2016.

34. Anuradha.C, Khanna.V, "A novel gsm based control for e-devices" Journal of Chemical and Pharmaceutical Sciences(JCPS) Volume 9 Issue 2, April - June 2016 .

35. Anuradha.C, Khanna.V, "Secured privacy preserving sharing and data integration in mobile web environments " Journal of Chemical and Pharmaceutical Sciences(JCPS) Volume 9 Issue 2, April - June 2016.

36. Sundarraj, B., Kaliyamurthie, K.P. Social network analysis for decisive the ultimate classification from the ensemble to boost accuracy rates 2016 International Journal of Pharmacy and Technology 8

37. Sundarraj, B., Kaliyamurthie, K.P. A content-based spam filtering approach victimisation artificial neural networks 2016International Journal of Pharmacy and Technology 83.

38. Sundarraj, B., Kaliyamurthie, K.P. Remote sensing imaging for satellite image segmentation 2016 International Journal of Pharmacy and Technology $8 \quad 3$.

39. Sivaraman, K., Senthil, M. Intuitive driver proxy control using artificial intelligence 2016 International Journal of Pharmacy and Technology 84.

40. Sivaraman, K., Kaliyamurthie, K.P. Cloud computing in mobile technology 2016 Journal of Chemical and Pharmaceutical Sciences 92.

41. Sivaraman, K., Khanna, V. Implementation of an extension for browser to detect vulnerable elements on web pages and avoid click jacking 2016 Journal of Chemical and Pharmaceutical Sciences 92.

\section{AUTHORS PROFILE}

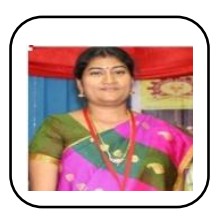

C.Anuradha, Assistant Professor, Department of Computer Science and Engineering, Bharath Institute of Higher Education and Research, Chennai.

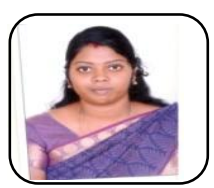

S.Sangeetha, Assistant Professor, Department of Computer Science and Engineering, Bharath Institute of Higher Education and Research, Chennai.

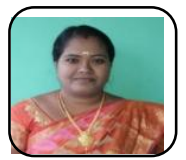

D. Vimala, Assistant Professor, Department of Computer Science and Engineering, Bharath Institute of Higher Education and Research, Chennai.

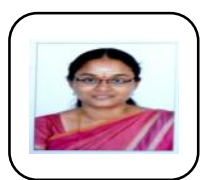

K. Shanmuga Priya, Assistant Professor, Department of Computer Science and Engineering, Bharath Institute of Higher Education and Research, Chennai. 